International Journal of Modern Physics C

(C) World Scientific Publishing Company

\title{
A qualitative study of a nanotube model using an iterative Taylor method
}

\author{
M. Gadella \\ Departamento de Física Teórica, Atómica y Óptica and IMUVA \\ Universidad de Valladolid, Paseo Belén 7 \\ Valladolid, 47011, Spain \\ manuelgadella1@gmail.com \\ L.P. Lara \\ Departamento de Física, FCEIA, UNR, Av. Pellegini 250 \\ 2000 Rosario, Argentina \\ lplara2014@gmail.com \\ J. Negro \\ Departamento de Física Teórica, Atómica y Óptica and IMUVA \\ Universidad de Valladolid, Paseo Belén 7 \\ Valladolid, 47011, Spain \\ jnegro@fta.uva.es \\ Received Day Month Year \\ Revised Day Month Year
}

Physical properties of graphene nanotubes may strongly depend on external fields. In a recent paper ${ }^{8}$, the authors have studied a model of carbon nanotubes under the presence of an external magnetic field, chosen for some symmetry properties. The model admits an exact solution, provided that the value of a parameter, here denoted as $k_{z}$, be equal to zero. This parameter is the eigenvalue of the component of the momentum in the direction of the nanotube axis. However, it seems that this parameter cannot be discarded for physical reasons. The choice of non-trivial values for this parameter produces an equation of motion for electrons in the nanotube (a Dirac-Weyl equation), which cannot be exactly solvable. Then, we proposed some iterative approximate methods to solve this equation and obtaining its eigenvalues. Some tests have shown that an iterative Taylor method that is more efficient than others proposed. For $k_{z} \neq 0$, we have found that, excluding the minimal energy eigenvalue, the lowest energy values obtained for $k_{z}=0$ split into two different ones and, therefore, producing gaps in the energy spectrum.

Keywords: Graphene nanotubes; Dirac-Weyl equation; iterative methods for ODE; iterative Taylor method; band structures.

PACS Nos.: 02.60.-x, 61.46.Np 


\section{Introduction}

In this paper, we intend to discuss some properties of carbon nanotubes of graphene in presence of external fields. Graphene is a material formed by a honey-comb lattice with carbon atoms at each vertex. This structure is an atom thick, so that it can be considered as two dimensional. Graphene is a material with some outstanding physical properties (strength, elasticity, conductivity of heat and electricity), that make it a candidate for multiple applications.

Single wall carbon nanotubes are cylindrical structures constructed as a result of bending graphene stripes. The radius may be orders of magnitude smaller than the length. Since the shape is cylindrical, any point on the nanotube surface is given by two variables, a length and an angle, the latter with appropriate periodic boundary conditions. As has been investigated, conducting properties of nanotubes may depend on the orientation of the atoms of the lattice in the cylinder and the radius. Thus, in relation to these two variables the energy band spectrum may or may not show a gap, so that nanotubes behave either as semiconductors or as conductors ${ }^{1}$. It is also very relevant that this gap, and therefore, the conducting properties may be altered by mechanical deformations and, which is more important from our point of view, by the presence of external fields.

Within this context, the energy band spectrum of carbon nanotubes in the presence of external of electric and/or magnetic fields has been largely discussed in the literature with different techniques, as for example the analysis of band structures in nanotubes with tight-binding Hamiltonians ${ }^{2,3,4}$. In another work, the authors show how conducting nanotubes may be turned into semiconducting, and conversely, by the application of an external homogeneous magnetic field, parallel to the nanotube axis $^{5}$. In another paper, it has been analyzed the effect of a homogeneous transverse field, in the low energy approximation, on the band spectrum ${ }^{6}$.

Thus, the study of the influence of external fields on carbon nanotubes becomes a very relevant research on this field. In the present paper, we consider a magnetic field which is constant in the direction parallel to the axis of the nanotube, the longitudinal direction, having an inhomogeneous transverse component, i.e., perpendicular to the axis. Our magnetic field has translational symmetry in one direction, a property already considered in the scientific literature ${ }^{7}$. This translational symmetry is a realistic and rather common situation, which simplifies the mathematical problem to be treated.

In a recent article $^{8}$, Jakubský et al. have discussed an exactly solvable model in which a transverse magnetic field interacts with the electrons in a single-wall nanotube. After the choice of the interaction ${ }^{8}$, the exact solvability was achieved by fixing one parameter equal to zero. However, it became clear that for other values of the same parameter there were not exact solvability and then approximate and numerical methods should be used. This is objective of the present paper.

First of all, let us describe the model. We consider a carbon nanotube one atom thick, having the form of a cylinder of infinite heigh and radius $\rho_{0}$. On the nanotube, 
a magnetic field $\mathbf{B}$ acts with a vector potential $\mathbf{A}=A_{\phi} \mathbf{n}_{\phi}+A_{z} \mathbf{n}_{\mathbf{z}}$, where $\mathbf{n}_{\phi}$ and $\mathbf{n}_{\mathbf{z}}$ are the unit vectors tangent to the circumference and to the longitudinal direction. The component $A_{\phi}$ was assumed to vanish ${ }^{8}$, while $A_{z}$ is a function of the angle $\phi$ alone, $A_{z} \equiv A_{z}(\phi)$. Along the present article, we are keeping the same assumptions, were $A_{\phi}$ and $A_{z}(\phi)$ are given data.

The behavior of a single electron on the nanotube is governed by a Dirac type equation of the form ${ }^{8}$ :

$$
H \widetilde{\Psi}(z, \phi)=\left[\sigma_{1} \frac{i}{\rho_{0}} \partial_{\phi}-\sigma_{2}\left(i \partial_{z}+\frac{q}{c \hbar} A_{z}(\phi)\right)\right] \widetilde{\Psi}(z, \phi)=\epsilon \widetilde{\Psi}(z, \phi),
$$

where $\sigma_{1}$ and $\sigma_{2}$ are the $2 \times 2$ Pauli matrices ${ }^{9}, q, c$ and $\hbar$ are the electron charge, the speed of light in the vacuum and the Planck constant divided by $2 \pi$ respectively. In (1), $\epsilon=E /\left(v_{F} \hbar\right)$, where $E$ is the energy and $v_{F}$ is the Fermi velocity in the graphene whose value is $v_{F} \approx 10^{6} \mathrm{~m} / \mathrm{s}$.

Note that equation (1) is linear with indeterminates $\epsilon$ and $\widetilde{\Psi}(z, \phi)$. Thus, we should look for factorizable solutions on both variables $z$ and $\phi$. Since (1) is a Dirac equation, it has two components. Its solution in terms of the indeterminate $\widetilde{\Psi}(z, \phi)$ is given by

$$
\widetilde{\Psi}(z, \phi)=e^{i k_{z} z} \Psi(\phi), \quad \Psi(\phi)=\left(\begin{array}{c}
\psi_{+}(\phi) \\
\psi_{-}(\phi)
\end{array}\right) .
$$

The form of $\widetilde{\Psi}(z, \phi)$ in (2) requires an explanation. The components $A_{\phi}$ and $A_{z}$ of the vector potential do not depend on the $z$ coordinate. This means that the Dirac-Weyl interacting Hamiltonian (1) must commute with the generator of translations in the $z$-direction, $P_{z}=-i \hbar \partial_{z}$. Thus, we look for eigenfunctions of the Hamiltonian $H$ in (1), which are also eigenfunctions of $P_{z}$. Thus, the physical meaning of $k_{z}$ is the momentum of an electron along the $z$ direction, which is here constant (2). Precisely, we here consider the consequences of being $k_{z} \neq 0$ on the electron allowed energy levels.

After (2), equation (1) depends on the variable $\phi$ alone so that (1) is transformed into

$$
\left(i \sigma_{1} \partial_{\phi}+\left(\rho_{0} k_{z}-\frac{2 \pi \rho_{0}}{\Phi_{0}} A_{z}(\phi)\right) \sigma_{2}\right) \Psi(\phi)=\rho_{0} \epsilon \Psi(\phi), \quad \Phi_{0}:=\frac{2 \pi c \hbar}{q} .
$$

This is an equation with two components, $\psi_{+}(\phi)$ and $\psi_{-}(\phi)$ as in (2). The solvability of the model depends on the choice of the vector potential $A_{z}(\phi)$ as well as the value of the parameter $k_{z}$. This solvability is guaranteed ${ }^{8}$ if $k_{z}=0$ and

$$
A_{z}(\phi)=\rho_{0} B_{0} a(\phi, k)
$$


where,

$$
a(\phi, k)=\left(1+k^{\prime}\right) \frac{s n[(\phi+\pi / 2) K / \pi] \operatorname{cn}[(\phi+\pi / 2) K / \pi]}{d n[(\phi+\pi / 2) K / \pi]} .
$$

Here, $s n(x, k), c n(x, k)$ and $d n(x, k)$ are the Jacobi elliptic functions and $K$ is a function of $k$ given by the following elliptic integral ${ }^{10}$ :

$$
K(k):=\int_{0}^{\frac{\pi}{2}} \frac{d t}{1-k^{2} \sin ^{2} t} .
$$

The parameter $k \in[0,1]$ is called the modular parameter. Sometimes one also uses $k^{\prime}:=\sqrt{1-k^{2}}$. Note that (5) and the properties of the elliptic functions imply that $\lim _{k \mapsto 0} a(\phi, k)=\cos \phi$.

As mentioned earlier, equation (3) is exactly solvable for the particular case $k_{z}=0$. The given solution shows two energy bands with positive energy with one gap between them. The first energy band is finite while the second one is unbounded ${ }^{8}$. The purpose of this section is to analyze some aspects on the behavior of the energy bands when $k_{z} \neq 0$ using an iterative Taylor method to be described next. We have chosen this method once we did a large number of numerical experiments using other methods and concluded that this gave more precise results with less operational complications.

This paper is organized as follows: In the next section, we introduce the iterative Taylor method that we shall use in our discussion on the above model. In Section 3, we discuss another two iterative approximate methods for the differential equations of our interest and compare their efficiency with the iterative Taylor method. The better efficiency of the iterative Taylor method justifies its choice to analyze the problem under our consideration. Section 4 contains the main results concerning the behavior of the graphene nanotube under the action of the external magnetic field. A discussion on the convergence of the iterative Taylor method is presented in an Appendix.

\section{Iterative Taylor method}

Let us consider a system of two ordinary differential equations of the following form:

$$
y^{\prime}(x)=f_{\lambda}(x, y(x), z(x)), \quad z^{\prime}(x)=g_{\lambda}(x, y(x), z(x)),
$$

where the indetermined functions $y(x)$ and $z(x)$ are defined on a given interval $[0, p]$, with $p<\infty$. The tilde denotes derivation with respecto to $x$. The functions $f_{\lambda}$ and $g_{\lambda}$ are given data and depend on the variables $x, y, z$ and linearly on the eigenvalue $\lambda$. As functions of $x$, they do not have singular points on the open interval $(0, p)$.

Since our aim is the study of the nanotube model described in the Introduction, we require that the solutions of (7) satisfy some boundary conditions. Thus, we assume that 


$$
y(0)=\alpha y(p), \quad z(0)=\alpha z(p), \quad \alpha= \pm 1 .
$$

Solutions satisfying (8) with $\alpha=1$ or $\alpha=-1$ are called periodic or anti-periodic, respectively.

Up to our knowledge, this problem has no exact solution, so that we are looking for approximate solutions. To this end, we propose an iterative method which is based in the Taylor expansion ${ }^{11}$. This method is conceptually very simple and quite easy to apply in comparison with other approximations ${ }^{12,13,14}$ including those discussed in the next section. In our opinion, this fact could make the iterative Taylor method particularly attractive.

In this approach, we obtain segmentary approximate solutions by a Taylor expansion of the indeterminate functions $(y(x)$ and $z(x)$ in the case of equation (7)). Needless to say that this requires that the indeterminate functions be differentiable up to a given order. First of all, we choose the intervals $I_{k}$ as before. On each of the intervals $I_{k}$, let us use the Taylor theorem in order to approximate he functions $y(x)$ and $z(x)$ in (7) by

$$
y_{m}(x):=\sum_{j=0}^{m} \frac{1}{j !} y^{(j)}\left(x_{k}\right)\left(x-x_{k}\right)^{j}, \quad z_{m}(x):=\sum_{j=0}^{m} \frac{1}{j !} z^{(j)}\left(x_{k}\right)\left(x-x_{k}\right)^{j},
$$

where we have chosen $x_{0}:=0$. Here, $y^{(j)}(x)$ and $z^{(j)}(x)$ are the $j$-th derivatives of the functions $y(x)$ and $z(x)$. Their values at the points $x_{k}$ are to be determined via equation (7). For $x_{0}:=0$, we fix some initial values $u:=y(0)$ and $v:=z(0)$, so that for the first derivative, we have

$$
y^{\prime}(0)=f_{\lambda}(0, u, v), \quad z^{\prime}(0)=g_{\lambda}(0, u, v) .
$$

For the second derivative, we take into consideration that

$$
y^{(2)}(x)=\frac{\partial f_{\lambda}}{\partial x}+f_{\lambda} \frac{\partial f_{\lambda}}{\partial y}+g_{\lambda} \frac{\partial f_{\lambda}}{\partial z}, \quad z^{(2)}(x)=\frac{\partial g_{\lambda}}{\partial x}+f_{\lambda} \frac{\partial g_{\lambda}}{\partial y}+g_{\lambda} \frac{\partial g_{\lambda}}{\partial z},
$$

and proceed similarly for successive derivatives. The initial conditions $u$ and $v$ are not yet determined.

After (9), the approximate solution on $I_{1}$ for $y(x)$ is given by

$$
y_{m}(x)=u+y_{m}^{\prime}(0)(x-0)+\frac{1}{2} y^{\prime \prime}(0)(x-0)^{2}+\cdots+\frac{1}{m !} y_{m}^{(m)}(0)(x-0)^{m} .
$$

Same for $z(x)$ with $z_{m}(0)=v$. These functions defined on the first interval $I_{1}$ give the values $y_{m}\left(x_{1}\right)$ and $z_{m}\left(x_{1}\right)$. Following the same procedure, we use $y_{m}\left(x_{1}\right)$ and $z_{m}\left(x_{1}\right)$ as initial values for the solutions (9) on $I_{2}$ and so on. At the final step, we obtain $y_{m}\left(x_{n}\right)$ and $z_{m}\left(x_{n}\right)$, which have to depend on $u, v$ and $\lambda$. 
Next, we use boundary conditions (8) on the approximate solutions obtained as above. Then, $u$ and $v$ have to be chosen so that the solutions obey to the required parity. Once, $u$ and $v$ have been fixed, we obtain an equation solely on $\lambda$, which determines suitable values of the eigenvalue $\lambda$.

In the particular case in which $f_{\lambda}$ and $g_{\lambda}$ as in (7) are linear on $y$ and $z$, system (8) is linear and homogeneous on $u$ and $v$. Then, the determinant of the coefficient matrix, $\Delta$, vanishes. Under this linearity hypothesis, $\Delta=0$ is just an algebraic equation on $\lambda$. Its roots are an algebraic function of the parameters in (1). The values for $n$ (number of intervals) and $m$ (degree of the polynomials (9)) are fixed empirically in order to obtain the desired accuracy. The use of Mathematica is now an important tool in our calculations of both Taylor coefficients and values of $\lambda$.

\section{Other iterative methods}

Iterative methods based in segmentary integration seem to be appropriate to approximate solutions to systems like (7). Along the present section, we introduce two other methods, besides the iterative Taylor method. The first one is called the successive approximation method (SAM). The second one is called the matrix method , which is applicable to linear systems only, as is our case. Both SAM and the iterative Taylor method are also suitable for second order non-linear equations (or non-linear systems of the type (7)).

Then, a discussion about the comparison of the precision between these three methods will justify the use of the iterative Taylor method to our physical system.

\subsection{The successive approximation method (SAM)}

In here, we shall not intend to study equations (7) with boundary conditions (8) in their full generality. Due to the form of the problem under our consideration, we shall restrict ourselves to the linear case. Let us consider an equation of the form:

$$
y^{\prime \prime}(x)+(\lambda a(x)-V(x)) y(x)=0,
$$

where $a(x)$ and $V(x)$ are know functions. This is a particular case of (7), as we may see by choosing $z(x):=y^{\prime}(x)$. Then, both $y(x)$ and $y^{\prime}(x)$ should have identical boundary conditions.

Note that $\lambda$ is positive whenever $a(x)$ and $V(x)$ be positive. To show this, let us multiply (13) by $y(x)$ and then, integrate by parts the term $\int_{0}^{p} y^{\prime \prime}(x) y(x) d x$ taking into account the boundary conditions given by $(7)$ (with $y^{\prime}(x) \equiv z(x)$ ). This gives:

$$
\lambda=\frac{\int_{0}^{p}\left[y^{\prime 2}(x)+V(x) y^{2}(x)\right] d x}{\int_{0}^{p} a(x) y^{2}(x) d x},
$$

which proves our claim. 
Equation (14) suggest the following method of successive approximations: Take the initial values (that may be also looked as boundary conditions) $y(0)=1$ and $y^{\prime}(0)=0$ and an initial value for $\lambda$, say $\lambda_{0}$. Assume that we can solve (13) under these conditions. We obtain a solution called $y_{0}(x)$. Then, use this solution in (14) to obtain a new value for $\lambda$, that we shall denote by $\lambda_{1}$. With this value and using the same initial conditions, we solve (13) to obtain a solution, $y_{1}(x)$ and so on. The $k$-th iteration can be written as:

$$
\begin{array}{r}
y_{k}^{\prime \prime}(x)+\left(a(x) \lambda_{k}-V(x)\right) y_{k}(x)=0, \quad y_{k}(0)=1, \quad y_{k}^{\prime}(0)=0, \\
\lambda_{k+1}=\frac{\int_{0}^{p}\left(y_{k}^{\prime 2}(x)+V(x) y_{k}^{2}(x)\right) d x}{\int_{0}^{p} a(x) y_{k}^{2}(x) d x}, \quad k=0,1,2, \ldots, n .
\end{array}
$$

The number $n$ of iterations is usually determined by the desired precision on the evaluation of the eigenvalue $\lambda$. For instance, giving a $\delta>0$ such that $\mid \lambda_{k+1}-$ $\lambda_{k} \mid<\delta$ for $k$ sufficiently large. In general terms, we need numerical methods in our calculations.

We need a procedure to determine the value $\lambda_{0}$, which is usually named as the seed. Let us consider the equation

$$
y^{\prime \prime}(x)+\omega^{2} y(x)=0, \omega^{2}:=\langle a(x)\rangle \lambda_{0}-\langle V(x)\rangle ;\langle f(x)\rangle=\frac{1}{p} \int_{0}^{p} f(x) d x .
$$

Then, solve equation (16) under the given periodic boundary conditions for the solution. This gives the admissible values of $\omega$ and therefore for $\lambda_{0}$. For instance if we take $p=2 \pi$, we obtain $\omega_{j}=2 j$ with $j$ integer. Therefore, we have the following sequence of admissible seeds, labeled by the integer value $j$ :

$$
\lambda_{0, j}=\frac{4 j^{2}+\langle V(x)\rangle}{\langle a(x)\rangle} .
$$

This completes our presentation of the SAM.

\subsection{The Matrix method}

This first method can be applied to first order linear systems only ${ }^{14}$. In general, such a linear system has the form $W^{\prime}(x)=A_{\lambda}(x) W(x)$, where $W(x)$ is the column vector of the undetermined functions, $A_{\lambda}(x)$ a square matrix depending continuously on the variable $x$ and linearly on the parameter $\lambda$ and the prime denotes derivative with respect to $x$. System (7) can be written on this form with $W(x)=(y(x), z(x))^{T}$, where the superscript $T$ means transpose. Obviously, equation (3) may also be written in the same form.

We want to determine an approximate solution of the system $W^{\prime}(x)=$ $A_{\lambda}(x) W(x)$ using a segmentary procedure ${ }^{14}$. To implement it, we consider an initial 
value $W(0)=(u, v)^{T}$, where $u$ and $v$ are real number to be assigned. Then, divide the interval $(0, p)$ into equally spaced segments $I_{k+1}=\left(x_{k}, x_{k+1}\right)$, with $x_{k}=k h$, $h=p / n$ and $k=0,1,2, \ldots, n$. On each segment $I_{k}$ we use the approximation $A_{\lambda}(x) \approx A_{\lambda}\left(x_{k}\right)$.

Then, we proceed with the integration on $I_{1}=\left(0, x_{1}\right)$ of the system with constant coefficients $W^{\prime}(x)=A_{\lambda}(0) W(x)$. Its solution gives the approximate solution on the interval $I_{1}$ and the value of this solution at the point $x_{1}$ is $W\left(x_{1}\right)=\exp \left(A_{\lambda}(0) h\right) W(0)$. Similarly, using $W\left(x_{1}\right)$ as initial condition, we integrate $W^{\prime}(x)=A_{\lambda}\left(x_{1}\right) W(x)$ on $I_{2}=\left(x_{1}, x_{2}\right)$. Then, we repeat the process on each of the interval $I_{k}$, using the value $W\left(x_{k-1}\right)$ as initial condition. In the last step, we obtain

$$
W(p)=\prod_{k=0}^{n-1} \exp \left(A_{\lambda}\left(x_{k}\right) h\right) W(0) .
$$

In order to obtain the eigenvalue $\lambda$, also called the characteristic value, we make use of one of the periodic boundary conditions $W(p)=\alpha W(0), \alpha= \pm 1$. Then, we arrive to the eigenvalue equation

$$
B_{\lambda} W(0)=0, \quad \text { with } \quad B_{\lambda}=\prod_{k=0}^{n-1} \exp \left(A_{\lambda}\left(x_{k}\right) h\right)-\alpha I,
$$

where $I$ is the identity matrix.

Consider now, $\operatorname{det} B_{\lambda}=0$. This is an algebraic equation of order $n$, whose solutions, i.e., the values of $\lambda$ can be determined by the use of, say, Mathematica. Fix one of these values of $\lambda$; then, the eigenvalue equation in (19) gives $W(0)=(u, v)^{T}$ save for a multiplicative constant, so that it is always possible to choose $u=1$.

Then, one way to obtain $v$ and therefore the initial condition $W(0)$ for each eigenvalue $\lambda$ is the following: First of all, note that each component in (19) must be linear on $v$. Then, for the first component in (19), that we denote here as $\left(B_{\lambda} W(0)\right)_{1}=0$, we obtain a relation $v=v(\lambda)$. Once we have determined the initial condition for a given $\lambda$, we have obtained the approximate solution for $W(x)$.

A second operation valid to determine the solutions of the eigenvalue problem (19), and therefore the approximate solution to $W^{\prime}(x)=A_{\lambda}(x) W(x)$, goes in this way: From $\left(B_{\lambda} W(0)\right)_{1}=0$, derive the relation $v=v(\lambda)$. We still do not know the eigenvalues $\lambda$. To determine these $\lambda$ 's, let us consider the polynomial $Q(\lambda):=$ $\left(B_{\lambda} W(0)\right)_{2}$, where the subscript 2 denotes second component in the eigenvalue equation (19). The roots of $Q(\lambda)$ give the eigenvalues, hence the values of $v$ and the segmentary eigenfunction $W(x)$.

\subsubsection{Application via Riccati equation}

Let us consider a Schrödinger type equation, which can be written in the form 


$$
y^{\prime \prime}(x)+(\lambda-V(x)) y(x)=0,
$$

and let us define a new indeterminate as $w(x):=y^{\prime}(x) / y(x)$. This is the typical substitution that transforms a Schrödinger equation into an inhomogeneous Riccati equation:

$$
w^{\prime}(x)+w^{2}(x)=V(x)-\lambda,
$$

with the boundary condition $w(0)=w(p)$. Then, on each interval $I_{k}=\left(x_{k}, x_{k+1}\right)$, we approximate $V(x)-\lambda$ by $V\left(x_{k}\right)-\lambda=V_{k}-\lambda$ (this defines $V_{k}$ ). After this approximation, we can obtain a recursive solution (constant on each interval $I_{k}$ ) of equation (21), which is given on the interval $I_{k+1}$ by

$$
w_{k+1}=\sqrt{V_{k}-\lambda} \frac{w_{k}+\sqrt{V_{k}-\lambda} \tanh \left(h \sqrt{V_{k}-\lambda}\right)}{\sqrt{V_{k}-\lambda}+w_{k} \tanh \left(h \sqrt{V_{k}-\lambda}\right)},
$$

with $k=0,1, \ldots, n-1$. Taking into account the boundary condition, this shows that

$$
w(0)-w(p)=0 .
$$

This gives an equation on $\lambda$ whose solutions are the eigenvalues (characteristic values). Note that the dependence in $\lambda$ in equation (21) appears in $w(p)$ only, so that this equation depends on $w(0)$, which should be fixed conveniently for each particular situation investigated.

\subsection{Numerical comparison of the methods}

Our methods have been designed in order to apply them to calculations on graphene nanotubes. However, we would like to know which ones are the most effective in these calculations. Then, it seems natural to check the efficiency of these methods by using some test. Thus, as a laboratory to compare the efficiency and numerical accuracy of our method, we use here the Mathieu equation ${ }^{10}$. If we take as reference that particular case, we obtain equations with periodical coefficients and one possible approximation gives the Mathieu equation. It has the following form:

$$
y^{\prime \prime}(x)+(r-2 q \cos (2 x)) y(x)=0 .
$$

In (24), $q$ is a given parameter, while $r$ is the characteristic value or eigenvalue which should be determined. There are four series of periodic solutions of (24), each one labeled with a discrete series of characteristic values ${ }^{15}$. Here we choose even periodic solutions on the interval $[0,2 \pi]$, which are usually written in the form ${ }^{16,17}$ : 


$$
y\left(x, q, r_{2 m+1}(q)\right)=\sum_{k=0}^{\infty} A_{k} \cos (2 k+1) x, \quad m=0,1,2, \ldots .
$$

If we take for instance $q=1$, the three first characteristic values are ${ }^{10} r_{1}=$ 1.85911, $r_{3}=9.07837$ and $r_{5}=25.0209$. Once we have determined these three first characteristic values, let us define as customary the percentage relative error as

$$
\epsilon_{r} \%:=100\left|\frac{r_{\text {num }}-r_{\text {exact }}}{r_{\text {exact }}}\right|,
$$

where $r_{\text {num }}$ is the characteristic value obtained by a numerical procedure in contrast with its exact value $r_{\text {exact }}$. We have determined the cpu time, $t_{c p u}$ using the software Mathematica 9.0 and the hardware AMD Athol (tm) II X2 250 Processor with 4 GB RAM. All methods under our consideration require an initial value of the characteristic value, which we denote as $r^{*}$. This will be the seed for the SAM iterations or as the initial value for the calculation of the roots in the matrix method. Since the cpu time $t_{c p u}$ depends on the chosen seed, in order to compare the different cases we have taken $r^{*}:=1.05 r_{\text {exact }}$.

Thus, the numerical values we have obtained are the following:

1.- Successive Approximation Method. In the next table, we show the results obtained via SAM for the three first characteristics values and the percentage relative error in terms of the number $j$ of iterations:

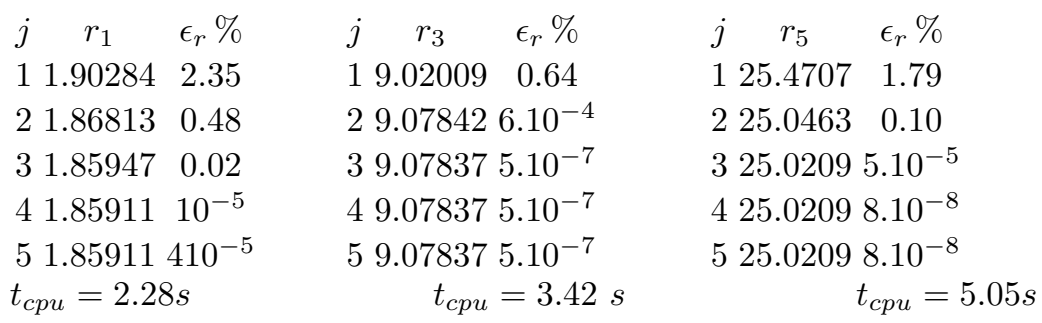

Here, cpu times grow due to the increase in the oscillations on $y$, which affects to the numerical evaluation of integrations.

2.- Matrix method (MM). Using this method, we show the results obtained, when we divide the interval $(0,2 \pi)$ into 10 and 12 subintervals, respectively (here and also in the following tables, $n$ denotes the number of intervals):

$$
\begin{aligned}
& \begin{array}{llllll}
k & r_{k} & \epsilon_{r} \% & k & r_{k} & \epsilon_{r} \%
\end{array}
\end{aligned}
$$

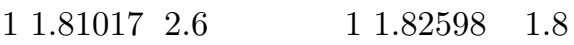

$$
\begin{aligned}
& \begin{array}{llllll}
3 & 9.0651 & 0.14 & 3 & 9.0707 & 9.10^{-2}
\end{array}
\end{aligned}
$$

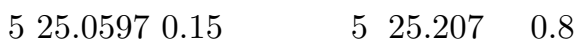

$$
\begin{aligned}
& t_{c p u}=15.9 \mathrm{~s} \quad n=10 \quad t_{c p u}=76.1 \mathrm{~s} \quad n=12
\end{aligned}
$$


Now the $t_{c p u}$ are too high due to the calculation of the roots. In order to improve these results, we rewrite the Mathieu equation in the Riccati form. Since we are looking for evan solutions, we take $w(0)=1$. Now we obtain a much better result as can be seen in the next table:

$$
\begin{aligned}
& \begin{array}{llllllllll}
k & r_{k} & \epsilon_{r} \% & k & r_{k} & \epsilon_{r} \% & k & r_{k} & \epsilon_{r} \%
\end{array} \\
& \begin{array}{llllll}
12.2399520 .5 & 11.85539 & 0.20 & 1 & 1.8584 & 4.10^{-2}
\end{array} \\
& 38.923471 .70 \quad 39.074285 .10^{-2} \quad 39.077687 .10^{-3} \\
& 524.84580 .70 \quad 525.018410^{-3} \quad 525.02051 .10^{-3} \\
& t_{c p u}=0.0654 \mathrm{~s}, n=10 \quad t_{c p u}=1.64 \mathrm{~s}, n=50 \quad t_{c p u}=7.21 \mathrm{~s}, n=100
\end{aligned}
$$

3.- Finally, we show the results obtained by the iterative Taylor method. Let us start with second order:

$$
\begin{aligned}
& \begin{array}{llllllllll}
k & r_{k} & \epsilon_{r} \% & k & r_{k} & \epsilon_{r} \% & k & r_{k} & \epsilon_{r} \%
\end{array}
\end{aligned}
$$

\begin{tabular}{|c|c|c|}
\hline$\epsilon_{r} \%$ & $\epsilon_{r} \%$ & $\epsilon_{r} \%$ \\
\hline $11.8572510^{-1}$ & $11.859173 .10^{-3}$ & $11.859112 .10^{-4}$ \\
\hline $\begin{array}{lll}3 & 8.6328 & 4.9\end{array}$ & $39.081363 .10^{-2}$ & $39.078572 .10^{-3}$ \\
\hline 520.475318 & $\begin{array}{lll}5 & 25.0932 & 0.29\end{array}$ & $525.02753 .10^{-2}$ \\
\hline
\end{tabular}

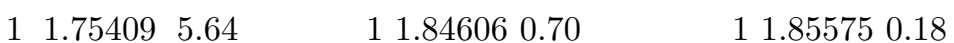

$$
\begin{aligned}
& \begin{array}{llllllll}
3 & 3.19332 & 65 . & 3 & 8.679 & 4.5 & 38.96382 & 1.2
\end{array} \\
& 50.19829599 . \quad 522.546610 . \quad 524.40152 .5 \\
& t_{c p u}=0.17 \mathrm{~s} \quad n=10 \quad t_{c p u}=1.28 \mathrm{~s} \quad n=50 \quad t_{c p u}=4.64 \mathrm{~s} \quad n=100
\end{aligned}
$$

Note that for second order, times $t_{c p u}$ are reasonable. However, we do not consider the accuracy as acceptable and therefore, we make the same numerical analysis using fourth order Taylor. We obtain:

From the precedent tables, we observe the following:

i.) The matrix method for Riccati has the same level of accuracy like the Taylor method with $n=100$.

ii.) Contrarily as it happens with the Taylor method, the matrix method in Riccati reduces the error when computing larger eigenvalues.

iii.) The SAM is more efficient for the Mathieu equation: we just need four iterations to obtain the eigenvalues $r_{1}, r_{3}$ and $r_{5}$ with better accuracy than in the other case and with $t_{c p u} \approx 10$ seconds.

Finally, in order to close our discussion, we perform the same calculations using the classical finite difference method, which approaches the second derivative of $y(x)$ evaluated at $x_{k}$ by its discrete derivative $h^{-2}\left(y_{k-1}-2 y_{k}+y_{k+1}\right)$ : 


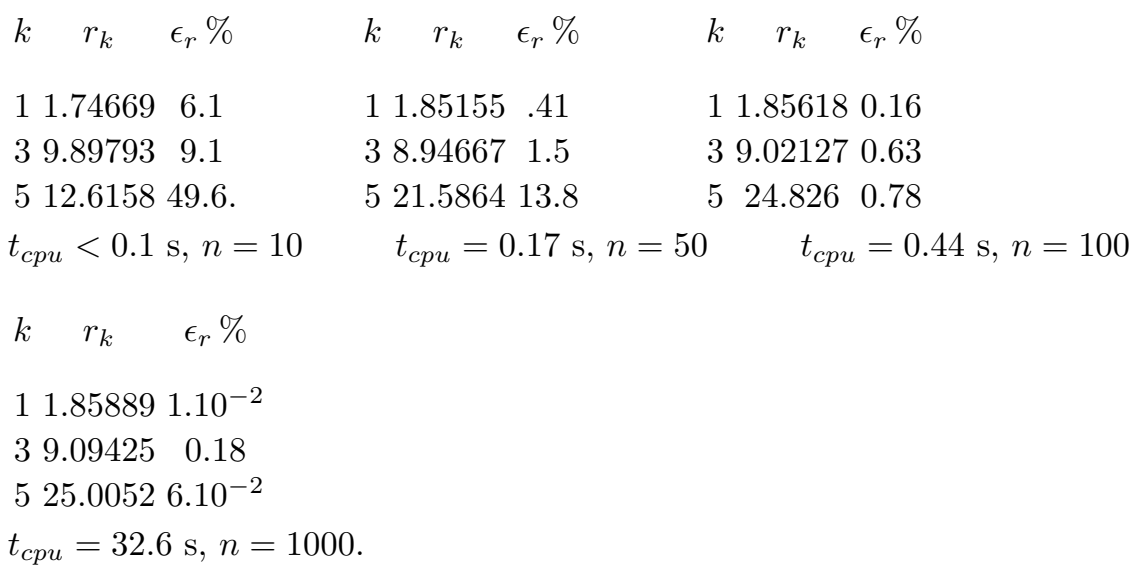

One should note that in this case, we need much higher values of $n$ in order to obtain a similar precision than for the previous methods, along with a non desirable significative increase of $\mathrm{cpu}$ times.

The conclusion is that the iterative Taylor method looks like more efficient than the others here proposed for the type of equation we want to solve.

\section{Discussion on the model of graphene nanotubes.}

Let us go back to the model as described in the Introduction. First of all, the value of $k$ in (5) varies from 0 to 1 and is fixed. In order to make the calculations with Mathematica easier, let us use instead the parameter $m$ defined as $m:=k^{2}$. Observe that in (5), this produces $\left(k^{\prime}\right)^{2}=1-m$. It is also convenient to define the variable

$$
x:=\frac{(\phi+\pi / 2) K(m)}{\pi},
$$

where $m$ is fixed so that $x$ depends solely on the angle $\phi$. Also, for constant $m$ the Jacobi functions depend only on the variable $x$, but in any case, we shall write $s n(x, m), c n(x, m)$ and $d n(x, m)$ for these Jacobi functions.

Then, we can write the two components matrix equation (3) as

$$
H \Psi(x)=\frac{\pi \rho_{0}}{K(m)} \epsilon \Psi(x), \quad \Psi(x)=\left(\begin{array}{c}
\varphi_{+}(x) \\
\varphi_{-}(x)
\end{array}\right),
$$

where $H$ has the following form:

$$
H=\left(\begin{array}{cc}
0 & i A^{+} \\
-i A^{-} & 0
\end{array}\right), \quad \text { with } \quad A^{ \pm}= \pm \partial_{x}+W(x) .
$$

Here, $\partial_{x}$ denotes derivative with respect to $x$ and 


$$
W(x)=-\frac{\pi \rho_{0}}{K(m)} k_{z}+W_{0}(x), \quad W_{0}(x)=2 m \frac{\operatorname{sn}(x, m) c n(x, m)}{d n(x, m)} .
$$

It is convenient to multiply (28) by $H$. It produces the effect of decoupling the system. This operation transforms (28) into

$$
H^{2} \Psi(x)=\left(\begin{array}{cc}
A^{+} A^{-} & 0 \\
0 & A^{-} A^{+}
\end{array}\right)\left(\begin{array}{c}
\varphi_{+}(x) \\
\varphi_{-}(x)
\end{array}\right)=\left(\frac{\pi \rho_{0}}{K(m)}\right)^{2} \epsilon^{2} \Psi(x) .
$$

Next, let us define

$$
H_{+}:=A^{+} A^{-}, \quad H_{-}:=A^{-} A^{+},
$$

so that

$$
H^{2}=\left(\begin{array}{cc}
H_{+} & 0 \\
0 & H_{-}
\end{array}\right), \quad H_{ \pm}=-\partial_{x}^{2}+V_{ \pm}(x)
$$

with

$$
V_{ \pm}(x):=W^{2}(x) \pm W^{\prime}(x) .
$$

At this point, it is in order to comment that the chosen potential $a(\phi, k)$ given in (5) is nothing else than the superpotential for the potential $V(\phi, k)=s n^{2}(\phi, k)$, which is, save for a constant, the well known Lamé potential ${ }^{18}$.

From (31) we readily obtain two second order separate equations, one for $\varphi_{+}(x)$ and the other for $\varphi_{-}(x)$. These are eigenvalue equations for $H_{+}$and $H_{-}$, respectively, which are equally valid to obtain approximate values for the eigenvalues $\epsilon$, which is the objective of the present analysis. To this end, let us use the eigenvalue equation for $H_{-}$:

$$
\partial_{x}^{2} \varphi_{-}(x)+\left(\left(\frac{\pi \rho_{0}}{K(m)}\right)^{2} \epsilon^{2}-V_{-}(x)\right) \varphi_{-}(x)=0 .
$$

We are studying properties of nanotubes in terms of the angle $\phi$. Therefore, functions depending on $\phi$ have to show periodicity properties in terms of this angle. It has been proven that these solutions should be either periodic or anti-periodic ${ }^{8}$. This periodicity properties have to be inherited by the functions $\varphi_{ \pm}(x)$. This comes from (28) and (29), which imply that $A^{-} \varphi(x)=s \varphi_{+}(x)$ and $A^{+} \varphi_{+}(x)=-s \varphi_{-}(x)$ with $s=i \pi \rho_{0} \epsilon / K(m)$. From the former of these equations and the boundary conditions for $\varphi_{-}(x)$, one gets $\varphi_{+}(0)= \pm \varphi_{+}(P)$. From the second and $A^{+}=\partial_{x}+W(x)$ and the previous boundary conditions, we get $\varphi_{+}^{\prime}(0)= \pm \varphi_{+}^{\prime}(P)$. After (27), the 
period is $P=2 K(m)$. In particular, solutions of (35) satisfy either periodicity or anti-periodicity properties:

$$
\varphi_{-}(x)=\alpha \varphi_{-}(x+P), \quad \varphi_{-}^{\prime}(x)=\alpha \varphi_{-}^{\prime}(x+P),
$$

where $\alpha=1$ and $\alpha=-1$, respectively.

Changes of scale usually help in simplifying calculations. In particular, we propose the following:

$$
x / P \longrightarrow x, \quad 2 \pi \rho_{0} \epsilon \longrightarrow \epsilon, \quad P^{2} V_{ \pm} \longrightarrow V_{ \pm} .
$$

Under this change of scale along a unit system such that $p=1$ and $\pi \rho_{0}=1$, equation (35) transforms into

$$
\partial_{x}^{2} \varphi_{-}(x)+\left(\epsilon^{2}-V_{-}(P x)\right) \varphi_{-}(x)=0 .
$$

With these new units, we can write $V_{-}(x)$ as:

$$
\begin{array}{r}
V_{-}(x)=4 k_{z}\left(k_{z}-\right. \\
\left.m \frac{s n(2 K(m) x, m) c n(2 K(m) x, m)}{d n(2 K(m) x, m)}\right) \\
+4 m K^{2}(m)\left(2 s n^{2}(2 K(m) x, m)-1\right) .
\end{array}
$$

Note that for $k_{z}=0,(39)$ is essentially the Lamé potential. A Schrödinger equation of the type $-d^{2} \phi(x) / d x^{2}+V(x) \phi(x)=\epsilon^{2} \phi(x)$, where $V(x)$ is the Lamé potential has analytic solution in terms of the elliptic Jacobi functions ${ }^{18}$.

Let us assume that $m<<1$, for simplicity. At first order, the potential $V_{-}(x)$ looks like

$$
V_{-}(x)=2 k_{z}\left(k_{z}-2 m \pi \sin (2 \pi x)\right)-m \pi^{2} \cos (2 \pi x),
$$

so that (38) becomes a Hill equation. Furthermore, if we choose $k_{z}=0$, we obtain the Mathieu equation. In Figure 1, we compare the exact and approximate potentials for small values of $m$ and $k_{z}=1$. We see that in the range of chosen values $m \leq 0.20$, both exact and approximate potentials are quite similar.

\subsection{An interesting approximation}

Before using the iterative Taylor method of Section 2 for a qualitative study of the case $k_{z} \neq 0$, let us introduce another simple approximation. To this end, let us consider the following equation:

$$
y^{\prime \prime}(x)+(a-f(x)) y(x)=0,
$$




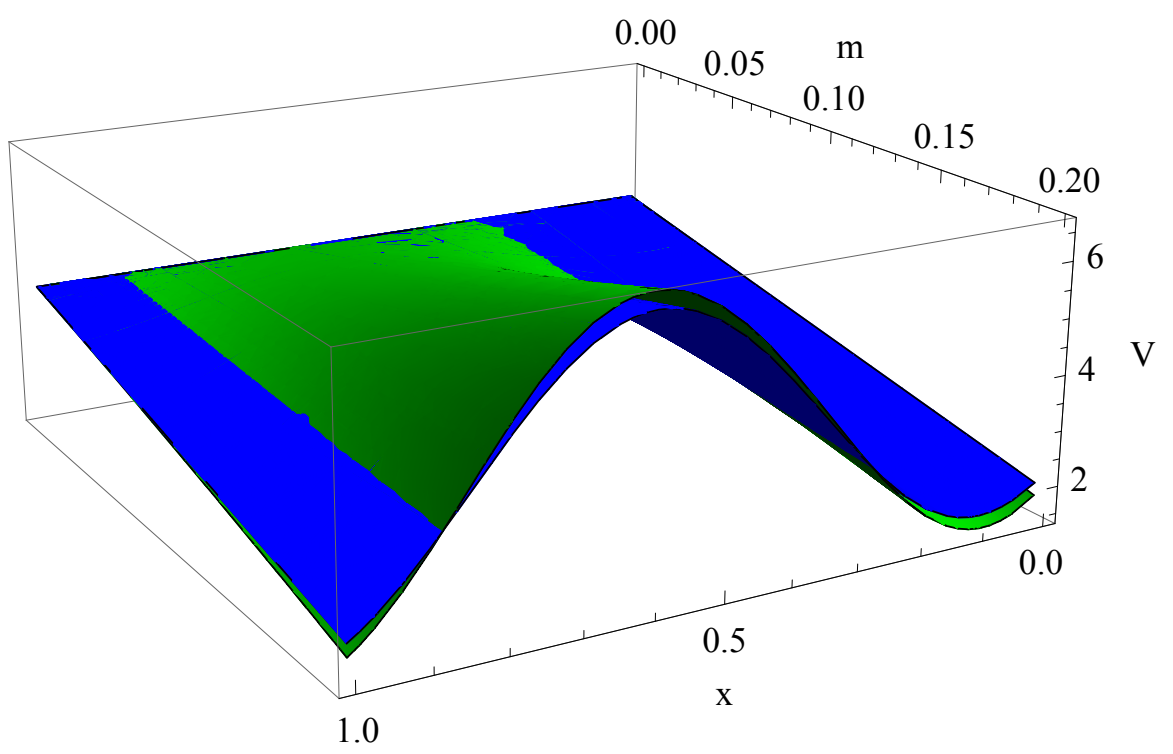

Fig. 1. Comparison between the exact potential (39) and the approximate (40), when $m<<1$ and $k_{z}=1$. The green and blue color correspond to the exact and approximate potentials respectively.

where $a$ is a fixed real number, $\alpha \leq x \leq \beta$ and $f(x)$ continuous on $(\alpha, \beta)$. Equation (41) can be approximated as

$$
\zeta^{\prime \prime}(x)+(a-\langle f(x)\rangle) \zeta(x)=0,
$$

where the meaning of the average $\langle f(x)\rangle$ is

$$
\langle f(x)\rangle:=\frac{1}{\beta-\alpha} \int_{\alpha}^{\beta} f(x) d x .
$$

In order to analyze the error in the approximation, let us consider the number $x^{*}$ such that $f\left(x^{*}\right)=\langle f(x)\rangle$. We know that $x^{*} \in(\alpha, \beta)$ due to the mean value theorem. Then, let us find the Taylor expansion of the solutions of (41) and (42) on a neighborhood of $x^{*}$. They are, respectively:

$$
\begin{aligned}
y(x) & =y\left(x^{*}\right)+y^{\prime}\left(x^{*}\right)\left(x-x^{*}\right)-\frac{1}{2}\left(a-f\left(x^{*}\right)\right) y\left(x^{*}\right)\left(x-x^{*}\right)^{2} \\
& -\frac{1}{6}\left(a-f\left(x^{*}\right)\right) y\left(x^{*}\right)\left(x-x^{*}\right)^{3}-\frac{1}{6} f^{\prime}\left(x^{*}\right)\left(x-x^{*}\right)^{3}+\ldots
\end{aligned}
$$

and 


$$
\begin{array}{r}
z(x)=z\left(x^{*}\right)+z^{\prime}\left(x^{*}\right)\left(x-x^{*}\right)-\frac{1}{2}(a-\langle f(x)\rangle) z\left(x^{*}\right)\left(x-x^{*}\right)^{2} \\
-\frac{1}{6}(a-\langle f(x)\rangle) z\left(x^{*}\right)\left(x-x^{*}\right)^{3}+\ldots .
\end{array}
$$

We see that (44) and (45) coincide up to second order. If we denote by $h$ the minimal radius of convergence of both series, we have that $h<\beta-\alpha$ (note that in the case of the graphene nanotubes we have chosen $\beta-\alpha=1$ ). Then, the error in the approximation is of the order of $h^{3}$.

Thus, equation (38) can be approximated by

$$
\partial_{x}^{2} \varphi_{a p p}(x)+\left(\epsilon^{2}-\left\langle V_{-}(P x)\right\rangle\right) \varphi_{a p p}(x)=0 .
$$

The function $\varphi_{a p p}(x)$ should approximate $\varphi_{-}(x)$. Then, the solution of (46) with either periodic and anti periodic boundary conditions on $\varphi_{a p p}(x)$ is trivial. When the boundary conditions are periodic, the energy values (eigenvalues) are

$$
\epsilon_{n}^{2} \approx(2 n)^{2} \pi^{2}+\left\langle V_{-}(P x)\right\rangle, \quad n=1,2,3, \ldots
$$

When the boundary conditions are anti periodic, we get the following energy values:

$$
\epsilon_{n}^{2} \approx(2 n+1)^{2} \pi^{2}+\left\langle V_{-}(P x)\right\rangle, \quad n=1,2,3, \ldots
$$

It is necessary to recall that in (38) the values of $\epsilon$ were rescaled. In order to obtain the true values we have to go back to the original scale. This approximation, although simple is a good one as we shall see by comparison with the results of the next subsection.

\subsection{Qualitative analysis based on the iterative Taylor method}

In this subsection, we introduce the results obtained after the use of the iterative Taylor method. Let us go back to equation (38). This, along boundary conditions, pose an eigenvalue problem for which the solutions give a discrete set of eigenvalues $\epsilon^{2}$. These eigenvalues are real and are customarily ordered as follows:

$$
\epsilon_{0}^{2}<\epsilon_{1}^{2} \leq \epsilon_{1^{\prime}}^{2}<\epsilon_{2}^{2} \leq \epsilon_{2^{\prime}}^{2}<\epsilon_{3}^{2} \leq \epsilon_{3^{\prime}}^{2}<\epsilon_{4}^{2} \ldots
$$

The eigenvalues with even subindices,

$$
\epsilon_{0}^{2}<\epsilon_{2}^{2} \leq \epsilon_{2^{\prime}}^{2}<\epsilon_{4}^{2}, \quad \text { etc }
$$


correspond to eigenvalues with periodic eigenfunctions. The eigenvalues with odd subindices,

$$
\epsilon_{1}^{2}<\epsilon_{3}^{2} \leq \epsilon_{3^{\prime}}^{2}<\epsilon_{5}^{2}, \quad \text { etc }
$$

correspond to anti-periodic solutions. The forbidden energy bands are:

$$
\left(\epsilon_{1}^{2}, \epsilon_{1^{\prime}}^{2}\right), \quad\left(\epsilon_{2}^{2}, \epsilon_{2^{\prime}}^{2}\right), \quad \text { etc },
$$

so that when $\epsilon_{k}^{2}=\epsilon_{k^{\prime}}^{2}$ the corresponding forbidden band disappears. On the other hand, the permitted bands are:

$$
\left[\epsilon_{0}^{2}, \epsilon_{1}^{2}\right], \quad\left[\epsilon_{1^{\prime}}^{2}, \epsilon_{2}^{2}\right], \quad \text { etc. }
$$

For the Lamé equation $\left(k_{z}=0\right)$, we know the following eigenvalues:

$$
\epsilon_{0}^{2}=0, \quad \epsilon_{1}^{2}=1-m, \quad \epsilon_{1^{\prime}}^{2}=1 .
$$

Their respective eigenfunctions are

$$
\psi_{0}(x)=\operatorname{dn} x, \quad \psi_{1}(x)=\operatorname{cn} x, \quad \psi_{1^{\prime}}(x)=\operatorname{sn} x,
$$

which shows that there exist two allowed and one forbidden bands ${ }^{8}$. When $k_{z} \neq 0$ there is no analytic solutions and therefore allowed and forbidden bands have to be determined by approximate methods such as the iterative Taylor or the simple method introduced in the previous subsection.

Let us go back to equation (38). When we used the iterative Taylor method, we have chosen a number of integration intervals $n=100$ for the Lamé equation. As we noted earlier, this equation has three exact eigenvalues to compare with. For the cases in which no exact solution is available, we have compared results with $n=100$ to results with $n=200$. We have obtained a relative variation of order less than $5 \cdot 10^{-2} \%$.

In Figure 2, we split $\epsilon_{1}^{2}$ and $\epsilon_{1}^{2}$ for increasing values of $m$, starting from $m=0$. Two curves that have smaller energy at $m=0$ correspond to $k_{z}=0$, i.e., the situation for which the exact solution is known. Here, the maximal error between the exact and numerical values is of the order of $5 \cdot 10^{-5} \%$. For the other two with higher energy at $m=0$, we have chosen $k_{z}=1$, so that no exact solution is known.

Figure 3 represents the variation of the numerical values of $\epsilon_{1}^{2}$ and $\epsilon_{1^{\prime}}^{2}$ with $\left|k_{z}\right|$, for a fixed value of $m$ that we have fixed here as $m=0.5$.

Going on with the same procedure, our numerical results show that the splitting of the levels other than the lower one is really small. To complete our analysis, let us go back to equations (47) and (48). Within the range $0<\left|k_{z}\right|<3$ and $0<m<0.7$, our results show a maximal relative difference, $\left|100\left(\epsilon_{\text {num }}^{2}-\epsilon_{\text {approx }}^{2}\right) / \epsilon_{\text {num }}^{2}\right|$, where 


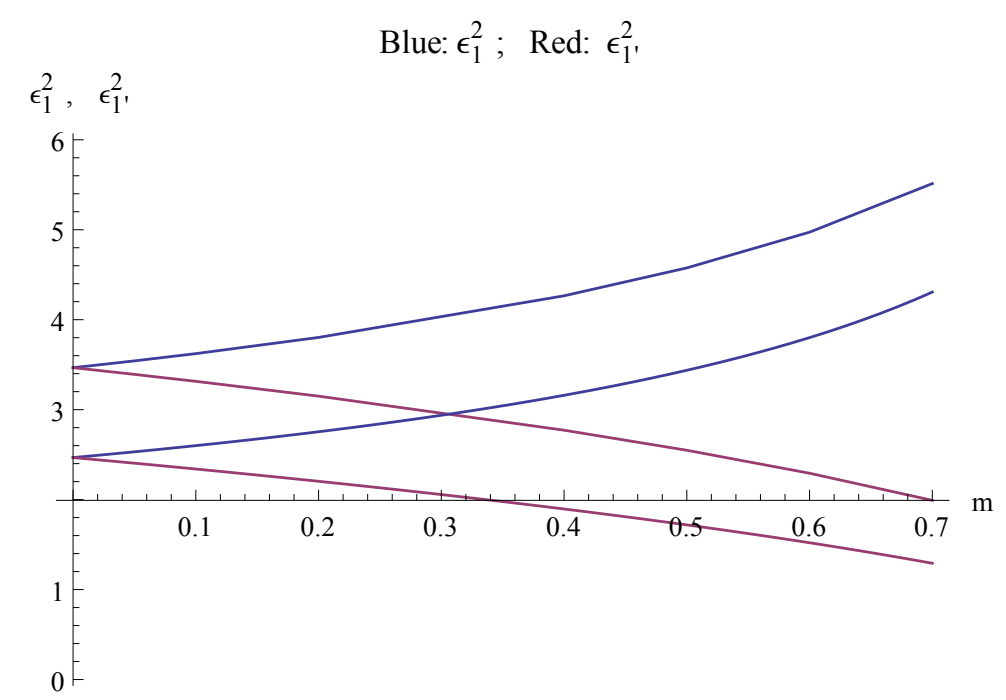

Fig. 2. Split between $\epsilon_{1}^{2}$ and $\epsilon_{1}^{2}$ for values $m>0$. The two lower lines corresponds to $k_{z}=0$ and the two upper to $k_{z}=1$.

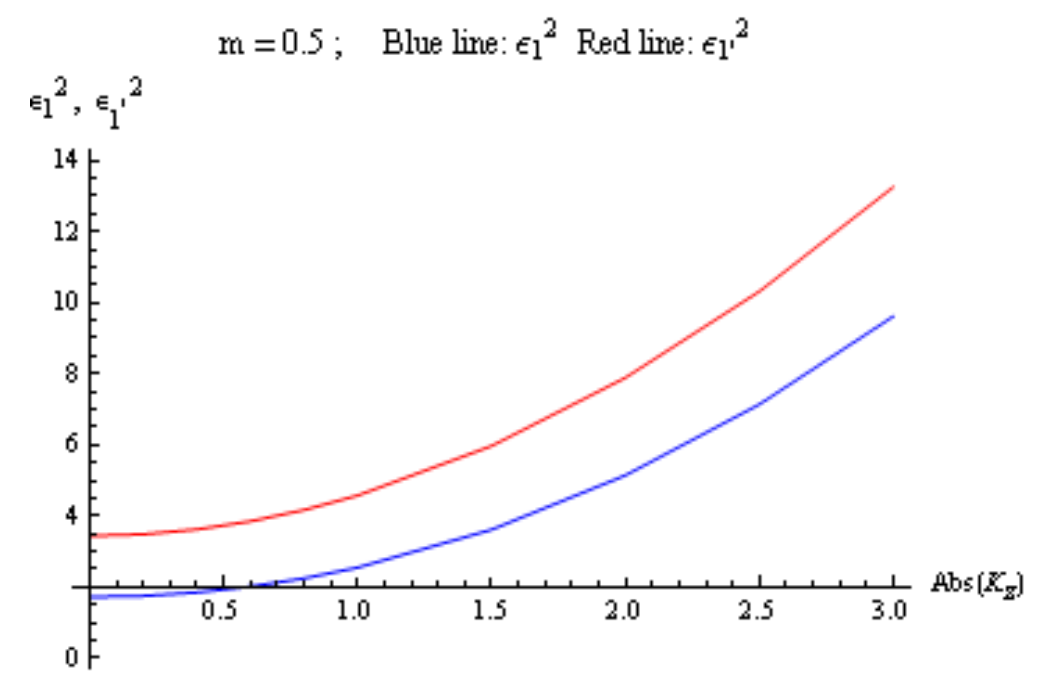

Fig. 3. Dependence of $\epsilon_{1}^{2}$ and $\epsilon_{1^{\prime}}^{2}$ with $\left|k_{z}\right|$ for $m=0.5$ fixed.

$\epsilon_{\text {num }}^{2}$ and $\epsilon_{\text {approx }}^{2}$ are the values obtained numerically and from the approximations given by formulas (47) and (48), respectively, of the order less than $0.2 \%$. This shows again a quite small splitting. Note this naive approximation is not really good for the ground level. In fact, $\left(\epsilon_{1}^{2}+\epsilon_{1^{\prime}}^{2}\right) / 2$ can be approximated by $\epsilon_{\text {approx }}^{2}$ with an error smaller than $1 \%$.

Finally, we have noted that the spectrum is very little sensitive to variations on 


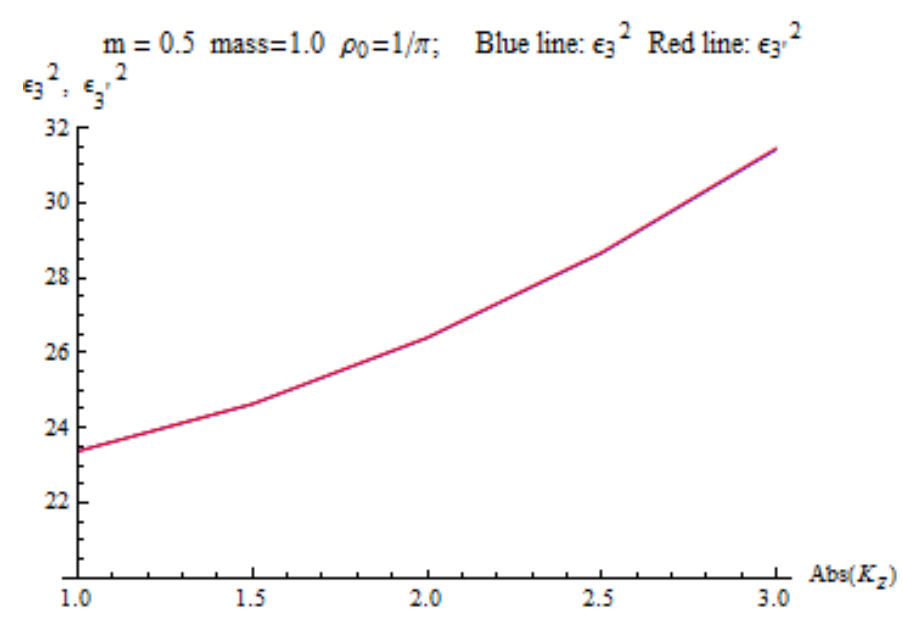

Fig. 4. Dependence of $\epsilon_{3}^{2}$ and $\epsilon_{3^{\prime}}^{2}$ with $\left|k_{z}\right|$ for $m=0.5$ fixed.

$m$ and $k_{z}$.

\section{Concluding remarks}

The structure of the energy bands and therefore, the physical properties of graphene nanotubes may depend on their interaction with external fields. In this paper, we complete the results of a previous work in which a single wall graphene nanotube was exposed to a magnetic field, which is constant in the direction of the nanotube axis and inhomogeneous in the direction perpendicular to this axis.

The explicit form of the magnetic field was chosen under two criteria: to have certain symmetry properties and to be exactly solvable. However, exact solvability was achieved if a parameter, $k_{z}$, which appears naturally in the formalism was taken equal to zero. Here, solvability means that the Dirac equation obtained with the given potential vector gives a analytic solution with either periodic or anti periodic boundary conditions.

However, there is no physics reason why this parameter (the eigenvalue of the momentum operator along the direction of the nanotube axis) should be zero. Then, it is interesting to study what the situation is if we give values to $k_{z} \neq 0$. Since the Dirac equation is an eigenvalue equation, our goal was to check whether the introduction of $k_{z} \neq 0$ produces a splitting of these eigenvalues and therefore, the presence of energy bands which did not appear for $k_{z}=0$.

Then the Dirac equation ceases to be exactly solvable and we need to use approximate methods for solution in order to obtain qualitative results. Since approximate iterative methods looked like suitable for this kind of problems, we have tested three of them in order to choose the most efficient one. The test was done on the Mathieu equation, in which we may compare approximate and exact solutions. These tests include numerical comparisons. Our conclusion was that the iterative Taylor 
method was the most efficient.

Our conclusion is that there is an effective split of eigenvalues other than the minimum and therefore, the appearance of permitted and forbidden energy bands. The forbidden energy bands corresponds to energies in between the energy split. For instance, the second energy eigenvalue splits for $k_{z} \neq 0$ in $\epsilon_{1}$ and $\epsilon_{1^{\prime}}$. Then, the energy gap lies in the interval $\left(\epsilon_{1}, \epsilon_{1^{\prime}}\right)$.

Our results show a clear split for the second energy eigenvalue, a small split for the third and a quite small split for the fourth that can be detected only with high precision. We can only conjecture the split of higher eigenvalues after our numerical tests.

One of the objectives of a future work may be to develop more efficient algorithms and methods to analyze higher eigenvalues as well as to apply our methods to other physical systems.

\section{Acknowledgements}

Partial financing support is acknowledged to the Spanish MINECO (Project MTM2014-57129), the Junta de Castilla y León (Project VA057U16) and the Project ING 19/ i 402 of the Universidad Nacional de Rosario.

\section{Appendix A. On the problem of the uniform convergence for the iterative Taylor method}

The iterative Taylor method consists in provide segmentary approximate solutions as in (9). We want to discuss when we can guarantee that these segmentary approximate solutions converge uniformly to the exact solution for equations of the form $y^{\prime}(x)=f(x, y(x))$, where $f(x, y)$ satisfy a Lipschitz condition with respect to the $y$ variable. Due to the form of system (7), this should be sufficient in the present case. This discussion is rather tedious and long although straightforward, so that we have it considered convenient to give it in an Appendix.

Assume that we divide the interval of integration on $n$ intervals. Then, the approximate segmentary solution is (see (9))

$$
T_{n}(x):=\left\{t_{n, k}(x), k=1,2, \ldots, n\right\},
$$

where $t_{n, k}(x)$ is the polynomial for the interval $I_{k}$. Since we want that $T_{n}(x)$ be an approximation for the solution of $y^{\prime}(x)=f(x, y(x))$, we should have

$$
\begin{gathered}
T_{n}^{\prime}(x)=f\left(x, T_{n}(x)\right)+\eta_{n}(x), \\
\eta_{n}(x)=T_{n}^{\prime}(x)-f\left(x, T_{n}(x)\right),
\end{gathered}
$$

where $\eta_{n}(x)$ appears due to the discrepancy between the exact solution and the approximate solution $T_{n}(x)$. An obvious integration on (A.2) gives 


$$
T_{n}(x)=y_{0}+\int_{x_{0}}^{x} f\left(t, T_{n}(t)\right) d t+\int_{x_{0}}^{x} \eta_{n}(t) d t,
$$

where $y_{0}$ is some initial condition. The point $x$ is arbitrary in the integration interval, $x \in\left(x_{0}, p\right], p=x_{n}$, so that it belongs to one of the subintervals, say $x \in I_{K}$. After (A.1) and (A.2), we can write

$$
\begin{gathered}
\int_{x_{0}}^{x} \eta_{n}(t) d t=\int_{x_{0}}^{x}\left[T_{n}^{\prime}(x)-f\left(t, T_{n}(t)\right)\right] d t \\
=\sum_{k=1}^{K-1} \int_{x_{k}}^{x_{k+1}}\left[t_{n, k+1}^{\prime}(t)-f\left(t, t_{n, k+1}(t)\right)\right] d t \\
\quad+\int_{K-1}^{x}\left[t_{n, k+1}^{\prime}(t)-f\left(t, t_{n, k+1}(t)\right)\right] d t .
\end{gathered}
$$

Then, taking (9) into account, we arrive to the following expression:

$$
\int_{x_{0}}^{x} \eta_{n}(t) d t=\sum_{k=1}^{K-1}\left[h_{n} a_{n, k+1}-\int_{k}^{x_{k+1}} f\left(t, t_{n, k+1}(t)\right) d t\right]+L T,
$$

where $L T$ is the last term in (A.4). Here,

$$
a_{n, k+1}=\sum_{j=1}^{m} \frac{1}{j !} y^{(j)}\left(x_{k+1}\right) h_{n}^{j-1}
$$

and $h_{n}$ is the length of each subinterval. Observe that the last term in (A.4) is indeed similar to the other with the only difference that $h_{n}$ should be replaced by a number smaller or equal. Now after (A.3), we have that

$$
\left|T_{n+m}(x)-T_{n}(x)\right| \leq \int_{x_{0}}^{x}\left|f\left(t, T_{n+m}(t)\right)-f\left(t, T_{n}(t)\right)\right| d t+\varepsilon_{n m},
$$

with

$$
\varepsilon_{n, m}=\left|\int_{x_{0}}^{x}\left[\eta_{n+m}(t)-\eta_{n}(t)\right] d t\right| .
$$

Let us use the Lipschitz condition in (A.7). This gives:

$$
\left|T_{n+m}(x)-T_{n}(x)\right| \leq R \int_{x_{0}}^{x}\left|T_{n+m}(t)-T_{n}(t)\right| d t+\varepsilon_{n m},
$$

where $R$ is a constant. Each of the functions in the sequence $\left\{T_{n}(x)\right\}$ is bounded, so that after (A.9) we obtain 


$$
\sup _{x \in\left[x_{0}, p\right]}\left|T_{n+m}(x)-T_{n}(x)\right| \leq \frac{\max \varepsilon_{n, m}}{\left|1-R\left(x-x_{0}\right)\right|}
$$

It is important to mention that $T_{n+m}(x)$ and $T_{n}(x)$ correspond to two different partitions of the integration interval. In these two different cases the partition has $n+m$ and $n$ subintervals, respectively. We shall denote by $\mathcal{P}_{n}$ to the partition with $n$ intervals. Without loss of generality, we may assume that $n=2^{p}$, where $p$ is a natural number, so that $\mathcal{P}_{m}$ with $m>n$ is always a refinement of $\mathcal{P}_{n}$.

In order to study the term $\varepsilon_{n+m}$, let us go back to (A.5) and consider

$$
\begin{array}{r}
\int_{x_{0}}^{x}\left[\eta_{n+m}(t)-\eta_{n}(t)\right] d t=\sum_{k=1}^{K_{(m+n)}-1} h_{n+m} a_{n+m, k}-\sum_{k=1}^{K_{(m)}-1} h_{n} a_{n, k} \\
+\sum_{k=1}^{K_{(m)}-1} \int_{x_{k}}^{x_{k+1}} f\left(t, t_{n, k+1}(t)\right) d t-\sum_{k=1}^{K_{(m+n)}-1} \int_{x_{k}}^{x_{k+1}} f\left(t, t_{n+m, k+1}(t)\right) d t \\
+L T_{(n+m)}-L T_{(n)} .
\end{array}
$$

In (A.11), we have used the symbol $K_{(p)}$ in the upper limit of the sum. This means that we have used the partition $\mathcal{P}_{p}$. The meaning of the two last terms should be obvious. Since we have chosen $\mathcal{P}_{n+m}$ to be a refinement of the partition $\mathcal{P}_{n}$, we may stand on the former. Then, using the definition (A.8), we have the following inequality

$$
\begin{array}{r}
\max \varepsilon_{n, m} \leq\left[\sum_{k=1}^{K_{(m+n)}} \max \left|a_{n+m, k}-a_{n, k}\right|\right] h_{n+m} \\
+\sum_{k=1}^{K_{(m+n)}} \int_{x_{k}}^{x_{k+1}}\left|f\left(t, t_{n, k+1}(t)\right)-f\left(t, t_{n+m, k+1}(t)\right)\right| d t .
\end{array}
$$

In (A.12) we have included the two last terms in (A.11). Due to their definition, the coefficients $a_{p, k}$ are uniformly bounded for any partition $\mathcal{P}_{p}$. The polynomials $t_{n, k}(t)$ and $t_{n+m, k}(t)$ have the same degree so that on each of the subintervals $I_{n+m}$, one has

$$
\left|t_{n, k}(t)-t_{n+m, k}(t)\right| \leq \alpha_{n, m, k} h_{n+m}
$$

with $\alpha_{n, m, k} \longmapsto 0$ when $n, m \longmapsto \infty$. In addition, the functions $T_{n}(x)$ and $T_{n+m}(x)$ are continuous on the same finite interval, so that the $\alpha_{n, m, k}$ are uniformly bounded by some $\alpha$. Using the Lipschitz condition for the function $f(x, y)$ with constant $R$ 
and (A.13) and taking into account that $h_{r}=p / r$, the term with the integral in (A.12) is bounded by

$$
\begin{array}{r}
\sum_{k=1}^{K_{(m+n)}} \int_{x_{k}}^{x_{k+1}}\left|f\left(t, t_{n, k+1}(t)\right)-f\left(t, t_{n+m, k+1}(t)\right)\right| d t \\
\leq \sum_{k=1}^{K_{(m+n)}} \alpha R h_{n+m}^{2}=\alpha(n+m) R h_{n+m}^{2}=\alpha R \frac{p^{2}}{n+m} \longmapsto 0,
\end{array}
$$

as $n+m \longmapsto \infty$. Thus, the second term in (A.12) goes to zero.

Concerning the first term in (A.12). The derivatives $y^{(j)}(x), j=1,2, \ldots, s$, are all bounded on the integration interval, because of their continuity on a compact interval. Then taking into account the explicit form of the $a_{n, k}$ given in (A.6), it is not difficult to show that this first term in (A.12) also goes to zero as $n+m \longmapsto \infty$. In consequence,

$$
\lim _{n+m \mapsto \infty} \max \varepsilon_{n, m}=0 .
$$

Let us go back to (A.10) and note that this inequality does not guarantee the uniform convergence of the sequence $T_{n}(x)$ if there exists an $x \in\left[x_{0}, p\right]$ such that the denominator in the right hand side of (A.10) vanishes. However, uniform convergence is assured if the interval width is smaller than $R^{-1}$, since in this case no such an $x$ may exist.

If the width of the interval $\left[x_{0}, p\right]$ were larger than $R^{-1}$, in order to ensure uniform convergence, let us choose $p_{1}:<x_{0}+1 / R$ and apply the procedure described on Section 2 to this interval. Then, repeat the method on the interval $\left[p_{1}, p_{2}\right]$ with $p_{2}<p_{1}+1 / R$ and so on.

We have shown that the sequence of approximate solutions $\left\{T_{n}(x)\right\}$ converges uniformly to a function $T(x)$. Then, using (A.14), the properties of the functions involved in this relation and the Lebesgue theorem, we conclude that

$$
T(x)=y_{0}+\int_{x_{0}}^{x} f(t, T(t)) d t
$$

so that $T(x)$ is the solution of $y^{\prime}(x)=f(x, y(x))$ with initial value $y\left(x_{0}\right)=y_{0}$.

\section{References}

1. J.C. Charlier, X Blase and S. Roche, Electronic and transport properties of nanotubes, Rev. Mod. Phys., 79, 677-732 (2007).

2. T.S. Li, M.F. Lin, Electronic properties of carbon nanotubes under external fields, Phys. Rev B, 73, 075432 (2006).

3. Y.H. Kim, K.J. Chang, Subband mixing rules in circumferentially perturbed carbon nanotubes: Effects of transverse electric fields, Phys. Rev. B, 64, 153404 (2001). 
24 M. Gadella \&S L.P. Lara \&S J. Negro

4. W. Degottardi, T.C. Wei, S Vishveshwara, Transverse-field-induced effects in carbon nanotubes, Phys. Rev B, 79, 205421 (2009).

5. S. Roche, G. Dresselhaus, M.S. Dresselhaus, R. Saito, Aharonov-Bohm spectral features and coherence lengths in carbon nanotubes, Phys. Rev. B, 62, 16092-16099 (2000).

6. H.W. Lee, D.S. Novikov, Supersymmetry in carbon nanotubes in a transverse magnetic field, Phys. Rev. B, 68155402 (2003).

7. F.M. Peeters, A. Matulis, Quantum structures created by inhomogeneous magnetic fields, Phys. Rev. B, 48, 15166-15174 (1993).

8. V. Jakubský, S. Kuru, J. Negro, Carbon Nanotubes in almost homogeneous transverse magnetic field: exactly solvable model, J. Phys A: Math. Theor., 47, 115307 (2014).

9. C. Cohen-Tannoudji, B. Diu, F. Laloë, Quantum Mechanics (Hermann, Paris, 1991).

10. M. Abramowitz, I. Stegun, Handbook of Mathematical Functions (Dover, New York, 1972).

11. H.T. Davis, Introduction to Non-linear Differential and Integral equations. (Dover, New York, 2010).

12. I.H. Abdel-Halim Hassan, On solving some eigenvalue problems by using a differential transformation, Appl. Math. Comp., 127, 1-22 (2002).

13. CK Chen, SH Ho, Application of Differential Transformation to Eigenvalue Problems, Appl. Math. Comp., 79, 173-188 (1996).

14. L. Lara, One-step recursive method for solving systems of differential equations, J. Comp. Appl. Math., 216, 210-216 (2008).

15. M. Farkas, Periodic Motions (Springer, New York, 1994).

16. G. Blanch, D.S. Clemm, The double points of Mathieu differential equation, Math. Comp., 23, 97-108 (1969).

17. M. Gadella, H. Giacomini, L.P. Lara, Periodic analytic approximate solutions for the Mathieu equation, Appl. Math. Comp., 271, 436-445 (2015).

18. F. Cooper, A. Khare, U. Sukhatme, Supersymmetry in Quantum Mechanics (World Scientific, London 2001). 